\title{
Mercosur-Tlcan-UE: triángulo atlántico o triángulo de las Bermudas
}

\author{
Jorge Grandi ${ }^{1}$
}

\section{Introducción}

Convergencia o divergencia, conflicto o cooperación, consenso o disenso son opuestos que coexisten en toda relación. Es complejo discernir y precisar donde termina la frontera de uno y donde se inicia la frontera del otro. En el caso de las relaciones entre países esta complejidad se multiplica y en las relaciones entre regiones mas o menos integradas se torna exponencial. Abordar la relación triangular entre el Mercosur, el Tratado de Libre Comercio de América del Norte (Tlcan) y la Unión Europea (UE) desde la perspectiva de los opuestos, es una tarea de largo aliento que solo puede plantearse un equipo multinacional y multidisciplinario. Fue con este criterio que a mediados de los 80 diversos estudios fueron encarados bajo el concepto de "Un Nuevo Triángulo Atlántico" (Grabendorff e Roett 1984). La perspectiva de estos análisis no fue el mismo en gran medida porque el desarrollo y el rol de la integración entonces no era el actual.

${ }^{1}$ Director, Centro de Formación para la Integración Regional (Cefir), Montevideo (Uruguay); Co-Director, Programa de Formación Unión Europea-Grupo de Río para la Integración Regional (FOR-CE RIO). Las opiniones expresadas son preliminares, se realizan a título personal y no comprometen a las del Centro en el que el autor presta sus servicios. 
Es al inicio de los sesenta, cuando la idea de algunos precursores del "Triángulo Atlántico" empieza a florecer apoyados en una amplia base conformada por: "Un sentimiento de conciencia occidental vincula a Europa, América del Norte y a muchas áreas de América Latina, y se expresa con múltiples vínculos: históricos, religiosos, políticos, económicos, militares y culturales." (Reidy 1964: 52). Pretender abordar en forma profunda este aun embrionario pero nada nuevo tema de debate en este primer ensayo, seria sumamente pretensioso. Es por ello que esta contribución se limita a esbozar y dar unas pinceladas de un "Nuevo Triángulo" que se está gestando a fines de los 90 y de imaginarlo como podría configurarse en la primer década del próximo siglo esperando que este se transforme en un próspero y pacífico triángulo y no en un mítico agujero negro como el "Triángulo de las Bermudas."

La relación entre los tres socios de este triángulo ha sufrido profundas transformaciones en las ultimas décadas. Nos detendremos en los tres vértices más dinámicos, el Mercosur, el Tlcan y la UE, y especialmente en el lado conformado por las relaciones entre el Tlcan y el Mercosur, eje en las negociaciones del Area de Libre Comercio de las Américas (Alca) y las del Mercosur-UE. Las relaciones de la nueva agenda transatlántica del norte entre Estados Unidos y la UE recientemente iniciadas serán tangencialmente abordadas (Krenzler e Wiegand 1999).

\section{La nueva dinámica de la integración de los años '90: Asunción-Madrid- Miami-Santiago de Chile}

Como es bien sabido por muchos, el Alca nace en diciembre de 1994, con motivo de la Cumbre de los 34 Presidentes americanos en Miami, sin embargo pocos recordaran que es la 1er reunión de este tipo en 27 años. La última realizada en 1967 en Punta del Este, se celebró, como es sabido, en un contexto político, económico e internacional radicalmente distinto: ausencia de democracia en muchos países de la región, influencia ascendente de las Doctrinas de Seguridad Nacional, fisuras en la adhesión a los principios de libre mercado, persistencia de la hegemonía mundial del dólar y del orden de Bretton Woods y como telón de fondo pero dominando casi todo el escenario la rivalidad de las dos grandes potencias y las consecuencias de los coletazos de la Guerra Fría en pleno apogeo en la región. Teledirigida desde el norte y contraria a aceptar cualquier iniciativa de integración originaria de la subregión, los resultados de esta Cumbre terminan confirmando y asegurando el fracaso de este encuentro y se acentúa a partir de ella un clima de divergencias, conflictos, disensos y desconfianza mutua. 
Muy distinto es el contexto, la perspectiva y la dinámica actual de esta primer Cumbre hemisférica de las Américas de Miami, donde un nuevo orden global aflora con perfiles muy propios a partir del inicio de los años ' 90 : fin de la guerra fría, hegemonía americana disputada en lo económico por la gravitante y renovada Europa del Mercado Unico, desmoronamiento de los regímenes de Europa Central y Oriental y creciente presencia económica de Japón y de los llamados tigres asiáticos. Un consenso de Washington que aunque hoy en su segunda rectificación menos extendido y menos compartido que el anterior ayuda a crear un "consenso" y una convergencia de políticas "pasiva para algunos" en todo la región sobre el rol del Estado y el mercado.

La afinidad de las ideas en términos generales, el clímax económico y político favorable y la necesidad de revitalizar la producción y el comercio impulsan, entre otros, el inicio de nuevo proceso de integración con una dinámica inédita en el Hemisferio.

Es en este contexto mundial que la Iniciativa para las Américas es lanzada por el entonces Presidente de Estados Unidos, George Bush, en junio de 1990. Desde la Doctrina Monroe en adelante, la historia hemisférica está cubierta de intentos estadounidenses de generar iniciativas, más o menos compulsivas, más o menos consensuadas según el prisma del observador, orientadas a buscar compromisos continentales.

Sin embargo, la apuesta a favor de la integración de Washington, del "nuevo regionalismo" es inédita y contradice la fuerte tradición estadounidense contraria a aceptar o secundar las iniciativas de integración que, ateniéndose al artículo 24 del Gatt, comenzaron a establecerse en los años ' 50 en todo el mundo, alcanzan en los 60 en Latinoamérica su primer apogeo y logran en los’ 90 su clímax más favorable a nivel mundial.

Es en este singular contexto internacional, y solamente un mes después del lanzamiento de la llamada Iniciativa Bush, en julio de 1990, que los Presidentes de Argentina y Brasil deciden dinamizar sustancialmente el proceso de integración entre ambos países abierto a mediado de los ' 80 . Ya se puede detectar, al inicio de los 90 con toda nitidez el decidido consenso existente en el Cono Sur de imprimir un impulso decisivo y vigoroso al por entonces algo alicaído proceso de integración y también, y no menos importante, la convicción de que este impulso debía verse acompañado de un fuerte aliento a las relaciones de la subregión con el resto del mundo, en particular Estados Unidos, el reciente y creciente Tlcan y una Comunidad Europea fortalecida por su Mercado Unico. La década perdida efectivamente llegaba 
a su fin y la revolución tranquila, al menos las energías de reforma que la motorizaban, se podían apreciar en esta fotografía del debut de la década.

En este nuevo marco de relaciones hemisféricas, que brinda el Alca que se reencuentran los dos bloques económicos más importantes en las Américas. El Tlcan y el Mercosur representan hoy el 95\% de la capacidad económica de las Américas, lo que los hace los dos centros de poder más significativos en las negociaciones. La interdependencia de estos dos bloques es acentuada e incremental y una crisis profunda en uno de ellos afectaría rápidamente al otro.

El salto cualitativo acordado entonces, julio de 1990, por los Presidentes Menem y Collor de Mello de acelerar los tiempos y generalizar la implementación en materia tarifaria y comercial de lo que ya entonces se comenzó a llamar Mercado Común es un hito paralelo al lanzamiento de la Iniciativa Bush. Como es sabido, el proceso de integración argentino-brasileño se ampliaría y adquiriría carta de naturaleza jurídica ocho meses después con la firma del Tratado de Asunción y el consiguiente nacimiento del Mercosur, en tanto emprendimiento subregional de integración (englobando en carácter de miembros plenos a Argentina, Brasil, Paraguay y Uruguay) más ambicioso en toda la historia del Cono Sur.

El Mercosur tuvo desde el primer momento entre sus objetivos centrales de proyección externa el establecimiento de relaciones estrechas con los grandes bloques económicos. La Unión Europea como principal socio comercial y fuente de inspiración del bloque fue una prioridad. Tan sólo algunos meses después de la suscripción del Tratado de Asunción, el 26 de marzo de 1991, se firma un Acuerdo Bilateral de Cooperación Inter-institucional.

El Comisario responsable de las relaciones con América Latina, Manuel Marín, anuncia el 19 de octubre de 1994 en Bruselas, un plan en dos fases encaminado a estrechar los lazos económicos y comerciales de la UE con el Mercosur. Un estudio de la Comisión Europea concluye que cuantificando los beneficios económicos que se derivan de ese escenario los intercambios se incrementarían a un ritmo del $11,5 \%$ anual, frente al actual 7\%. El documento de la Comisión fue aprobado por el Consejo Europeo de Essen de diciembre de 1994 y fue objeto de rondas de negociación entre funcionarios de la UE y del Mercosur en el transcurso de 1995, hasta lograr alumbrar un acuerdo innovador, denominado de "cuarta generación", que contempla un nuevo partenariado UE-Mercosur.

El Acuerdo de Cooperación Interregional, suscrito en Madrid el 15 de diciembre de 1995, establece el objetivo de reforzar la cooperación económica y comercial 
entre ambos bloques con el objetivo central de acometer una liberalización progresiva y recíproca de los intercambios que permita, en una segunda fase, lograr una zona de libre comercio, en cuya materialización habrá que esperar a que el Mercosur madure su condición de unión aduanera. Este objetivo superior del acuerdo podría alcanzarse a mediados de la próxima década y para ello se tendrían en cuenta la sensibilidad de ciertos productos.

A finales de marzo de 1996 en Buenos Aires se aprobó el reglamento de la Comisión Mixta UE-Mercosur que se reunió por primera vez en junio de 1996 en Bruselas. En dicha ocasión se firmó un Protocolo de Cooperación Aduanera entre la UE y el Mercosur. Este acuerdo tiene por objeto agilizar los trámites aduaneros a fin de favorecer el intercambio comercial entre los bloques y se perfilan nuevos ámbitos de cooperación. La Comisión Comercial prevista en el acuerdo se reunió por primera vez en marzo de 1997 y definió tres etapas para las negociaciones comerciales. En primer lugar, la realización de un diagnóstico sobre los intercambios de bienes entre ambos bloques que ya se ha culminado. En segundo lugar, la aproximación de posiciones con vistas al ulterior inicio de la liberalización de los intercambios a partir de entonces. En tercer lugar, la elaboración de propuestas concretas a ser elevadas a la Subcomisión Comercial (reunida por primera vez en Belo Horizonte en noviembre de 1996), a quien deben someter sus informes los tres Grupos de Trabajo creados: el de Bienes, el de Servicios y el de Disciplinas Comerciales. A partir de allí saldrán mandatos de negociación y según las previsiones actuales, habrá que esperar al año 2002 o 2003 para que maduren algunos acuerdos tendientes a liberalizar el comercio interregional.

El alcance de estos acuerdos hoy aparece incierto. En abril de 1997, el Consejo de Ministros de la UE aprobó una comunicación en la cual se recomienda la no suscripción por parte de la Unión de nuevos acuerdos de libre comercio, preconizándose por el momento el desarrollo de los acuerdos ya existentes.

Los intercambios comerciales UE-Mercosur se han duplicado en los últimos seis años, pasando de 24.000 millones de dólares en 1990 a 45.400 en 1996. Pero la intensidad de los flujos se ha modificado sustancialmente. Mientras las exportaciones del Mercosur a la UE crecieron sólo un 27,3\%, pasando de 17.400 a 22.120 millones de dólares, las exportaciones de la UE lo hicieron en un 246,5\%, pasando de 6.700 millones a 23.280 millones de dólares. Ello implica que la UE ha pasado de tener un déficit comercial de casi 11.000 millones de dólares en 1990 a tener un superávit de más de 1.000 millones de dólares en 1996. La UE representa en torno al 30\% del comercio exterior del Mercosur y es su primer socio comercial. La UE es el segundo 
inversor extranjero en la subregión, representando en torno al 35\% de la IED. Un $75 \%$ de las inversiones latinoamericanas de la UE se concentran en la subregión (un 70\% en el sector industrial). Los europeos han sido los primeros inversionistas en los procesos de privatizaciones emprendidos en los cuatro países (totalizando un $25 \%$ del conjunto de los capitales ofrecidos).

En suma, en poco más de seis años las relaciones del Mercosur con la UE han adquirido un dinamismo y una intensidad inéditas para el patrón de vinculación interregional predominante hasta entonces. Ello permite afirmar que seguirá siendo uno de los principales polos de atención latinoamericano en materia de integración de la Unión, un importante destinatario de la cooperación más avanzada y uno de los principales ámbitos de realización de emprendimientos conjuntos del sector privado europeo en regiones "emergentes".

Resulta interesante evidenciar el carácter estratégico de las relaciones comerciales del Mercosur con la UE que, de acuerdo a algunas estimaciones, supera con creces el horizonte de crecimiento del comercio que cabe esperar con los países del Nafta a partir de las negociaciones del Alca. Ello se desprende de un estudio de impacto de la Fundación Getulio Vargas dado a conocer a principios de 1997 y hecho en base a un escenario experimental de libre comercio para 37 tipos diferentes de bienes, que contrasta el impacto sobre las economías argentina y brasileña de una hipotética liberalización de los intercambios del Mercosur con ambos bloques. Según este estudio, en caso de producirse una genuina asociación comercial UEMercosur, el PIB de Argentina crecería un 6,71 \% y el de Brasil un 5,05\%, mientras que como consecuencia del Alca, lo haría tan sólo en un $0,68 \%$ y un $2,08 \%$, respectivamente. Por lo que respecta a los términos de intercambio, el estudio calcula para el primer escenario (zona de libre comercio UE-Mercosur) una mejora del 6,16\% para Argentina y un 3,59\% para Brasil, mientras que para el segundo (acuerdo Alca) la mejora resultante es de sólo 0,34\% para Argentina y 1,09\% para Brasil.

En las negociaciones que se espera comenzar a fines de junio próximo en Río entre el Mercousr y la UE en el marco de la Cumbre Presidencial América LatinaEuropa uno de los mayores desafíos que se enfrentan, además de que la Comisión obtenga el mandato del Consejo para negociar, es el tema agrícola. El reclamo del Mercosur para que se deroguen los subsidios europeos podría costarle a los agricultores perdidas anuales de 5 a 15 mil millones de dólares. El tema agrícola en al agenda futura 2000 europea esta siendo enérgicamente tratado al mas alto nivel. Estas negociaciones tienen un nivel prioritario y si se aceleran por una solución del problema agrícola podrán afectar negativamente el ritmo del Alca. 
Estados Unidos representa el socio comercial más importante de todos los países del Mercosur en el Hemisferio y el segundo a nivel mundial después de la UE. Y además, es el mayor inversor disputándole este puesto a la UE. Mercosur representa para los Estados Unidos el segundo socio en el Hemisferio después de México, le representa un gran mercado potencial y absorbe una gran parte de sus exportaciones mundiales y latinoamericanas (20\%), cualitativamente muy importante por su alto valor agregado. Por lo que respecta al comercio, cabe señalar que las exportaciones del Mercosur hacia EE.UU crecieron un 15\% en el período 199096, pasando de 12.230 a 14.100 millones de dólares, mientras las importaciones lo hicieron en un 195\%, pasando de 7.500 a 22.125 millones de dólares. En materia de inversiones, Estados Unidos acapara el 40\% de los flujos de IED.

En cuanto a las relaciones del otro importante socio del Tlcan, México con el Mercosur ellas han sido desde el origen conflictivas y llenas de desencuentros. Marcados por el no cumplimiento del conocido artículo 44 de Aladi que establece que si un país da privilegios a un tercero fuera de Aladi, como ha sido en el caso de México en el Tlcan, debe generalizar dichas ventajas a los demás miembros de la Asociación o compensar a sus socios. Las negociaciones no han tenido éxito hasta el momento entre otros por el escaso volumen de comercio entre los miembros del Mercosur y México, por el diferente nivel de preferencias otorgadas por México a los países miembros y por el grado de competencia de las exportaciones mexicanas a los Estados Unidos con los productos exportados por los miembros de Mercosur al mismo mercado.

La tradicional competencia de México y Brasil en el mercado americano de productos como jugo de naranja, zapatos y otros se inclinó positivamente del lado de México desde la conformación del Tlcan, siendo mucho menor el impacto negativo para Argentina y Uruguay. En este marco México acelera y mejora las relaciones en general a nivel bilateral con estos dos países, e incluso, en lo comercial Argentina ha recibido algunas compensaciones que provocaron alguna irritación con su socio mayor. A pesar de algunos escollos el esquema sigue siendo el sostenido por los países miembros en las relaciones Mercosur- México.

El la agenda de temas conflictivos de esta relación, entra el del Alca, ya que mientras México insiste en que son favorables a este proceso, otros opinan que no lo son al menos en el corto plazo y que la red de acuerdos de libre comercio que este país ha firmado con Chile, Colombia, Venezuela, Costa Rica, Bolivia y los países de América Central y que impulsa con otros países, así como con su iniciada tercera ronda de negociaciones entre México y la UE en 1999, en la que se espera resultado 
en las acuerdos relativos a un tratado de libre comercio antes de fin de año, confirman la estrategia de la bilateralidad. Los acuerdos ACE renovados en el marco de la Aladi con Argentina hasta el 2001, con Paraguay hasta este año y Uruguay hasta el 2000 también lo confirman.

México mantiene un superávit comercial con la subregión, sin embargo, el comercio es escaso en volumen y su cómoda posición producto de los acuerdos bilaterales fue interrumpida cuando Brasil prácticamente dejó sin efecto su acuerdo bilateral afectando rápidamente el comercio resultando en 1988 superavitario para Brasil. A partir de enero se iniciaron los esfuerzos de negociación entre Brasil y México pero esta se vió afectada por la crisis en Brasil.

Con respecto a las relaciones de Mercosur con el otro socio del Tlcan, Canadá, el Tratado de Libre Comercio sigue demorado. El tratado de ser firmado concede trato nacional y condición de nación más favorecida a cada miembro, lo que eliminaría barreras al comercio e incrementaría las inversiones. Brasil es el mayor socio de Canadá en el Mercosur pero solo representa un escaso $0,5 \%$ de su comercio total y muestra hoy indicios a la baja, sin embargo las principales exportaciones crecieron entre 1993 y 1997 52,2 \% y las de Brasil en un 151\%. Con Argentina en el mismo período el incremento de los rubros más importantes fue más sustantivo en términos relativos creciendo esas exportaciones argentinas 124,7\% y las canadienses $352,4 \%$. Con América Latina es el $2 \%$ y con USA el $82 \%$ de sus exportaciones en 1997 (Documentos Sela 1998).

El interés de Canadá es por lo tanto relativo e indirecto en el comercio con la subregión. Estados Unidos exporta 20\% de su comercio a América Latina, entonces una crítica situación en el Mercosur afectaría en forma tangencial su comercio con su socio más cercano. Desde la perspectiva de las inversiones la presencia canadiense se ha triplicado en los últimos años y se han diversificado al sector minero, telecomunicaciones, bienes de capital, motores de aeronaves y embarcaciones así como minería y equipos y servicios relacionados con la protección del medio ambiente, presentándose como rubros muy promisorios para este país en la subregión.

El gobierno del Primer ministro Chrétien ha intentado distanciarse en su política exterior de su vecino estadounidense, no solamente con respecto a Cuba en el que es un importante inversor y exportador y comprador. En sus visitas a los principales países del Mercosur realizado durante 1998 y en todos los foros del los 90 ha intentado modificar su identidad identificada tradicionalmente con la política norteamericana y orientada comercialmente hacia Europa, Asia y Estados Unidos. 
Canadá ha logrado en poco tiempo forjarse una identidad propia afirmada por una política destinada a expandir unilateralmente su presencia económica en América Latina. En el Marco de la OMC Canadá comparte con los países del Mercosur las mismas tesis en el capítulo agrícola y participa en el Grupo Cairns. Canadá preside estratégicamente la primera ronda de negociaciones del Alca y la próxima cumbre se realizará en este país.

En junio de 1995 comenzaron las negociaciones para la liberalización del comercio entre ambos bloques, aunque de hecho en los últimos dos años las relaciones Mercosur-Tlcan se han canalizado a través de las negociaciones que tienen lugar en el marco del Area de Libre Comercio de las Américas (Alca). En los tres primeros años del Mercosur, la expectativa de ampliación a corto plazo del Tlcan a países sudamericanos seleccionados produjo disensiones entre los dos grandes socios.

Mientras Argentina realizó esfuerzos en dicha dirección, Brasil mostró su preferencia en ampliar el Mercosur al resto de los países sudamericanos, proponiendo, a fines de 1993 la constitución en el plazo de diez años de un Área de Libre Comercio Sudamericana (Alcsa), proyecto que si bien no prosperó en las modalidades y en los plazos previstos por la Cancillería brasileña, puede afirmarse que actúa como referente de la negociación de una Zona de Libre Comercio entre el Mercosur y la CAN (Comunidad Andina de Naciones), iniciada en 1996 y teóricamente sustanciada en el Acuerdo suscrito en Buenos Aires en abril de 1998, pero cuyo calendario voluntarista de realización está sufriendo algunos escollos.

Un bloque mayor que dé contrapeso para equilibrar posiciones, aunque sea más político que económico en las negociaciones hemisféricas tendría efectos positivos para los dos subbloques. Ello es percibido, en ocasiones, como una maniobra de Brasil para utilizar un frente de países a su favor en las negociaciones hemisféricas.

Entre otros problemas en las negociaciones, más de orden comercial, se puede subrayar la complejidad técnica de acordar, consolidar, armonizar o alterar las preferencias existentes entre cada uno de los cuatro países del Mercosur y cada uno de los cinco países de la CAN, disimilitud de los enfoques y posturas de ambos bloques, discrepancias en el interior de cada bloque sobre los objetivos de tipo comercial y de otros órdenes que animan la estrategia de constitución de una zona de libre comercio bloque a bloque, disciplinas y tratamientos internos disímiles al interior de cada bloque a los países de menos desarrollo relativo. Uruguay y Paraguay en el Mercosur tienen algunos plazos adicionales de gracia para la adopción de determinadas políticas del bloque, en tanto Ecuador y Bolivia, país 
este último cuyo status se torna más complejo al ser país asociado del Mercosur, tienen decididamente prerrogativas y trato nacional a determinados sectores.

Los ritmos de aceleración o desaceleración en las negociaciones de los dos subloques parecen estar estrechamente relacionados con los ritmos del Alca, lo que en si no es positivo ya que el factor tiempo es clave para fortalecer los lazos y tener un frente común más sólido basado en interdependencias económicas comerciales más estrechas que las actuales.

Los trabajos en el ámbito técnico y ministerial del Alca tras la Cumbre de las Américas de diciembre de 1994 se desarrollaron en un ambiente internacional menos favorable a las tendencias de regionalización. Las conclusiones relativamente exitosas de la Ronda Uruguay del Gatt alientan lo multilateral, se presentan dificultades de la UE en su profundización, el Congreso de Estados Unidos rechaza el fast track y el déficit con el comercio con México se empieza a notar. El objetivo de crear una zona de libre comercio americana se mantuvo y comenzaron en 1995. Se crearon 11 grupos de trabajo -posteriormente ampliados a cuatro grupos consultivos adicionales- y se mantuvieron reuniones preparatorias de alto nivel en Denver en 1995, en Cartagena de Indias en 1996 y en Belo Horizonte (Brasil) en 1997 para después dar el puntapié inicial a las negociaciones en la II Cumbre de las Américas celebrada en Santiago de Chile en abril de 1998 que comenzaron formalmente en septiembre de este año en Miami.

Resulta de interés detenerse en la reunión de Belo Horizonte, ya que ella resulta trascendental no sólo por los acuerdos que permite alcanzar de cara a la II reunión Cumbre del Alca -la celebrada en Santiago de Chile- sino por el alto grado de consenso que consigue cristalizar entre los países del Mercosur en materia de posición negociadora común y unívoca. De acuerdo a lo trascendido públicamente y recogido a través de contactos con algunos de los funcionarios participantes, Belo Horizonte simboliza para el Mercosur un hito en la consecución de una única voz a través de una representación unitaria de defensa de los intereses del Mercosur en la negociación del Alca, a saber, la firme postura favorable a que la Zona de Libre Comercio Americana deberá ser el resultado de la convergencia de múltiples esquemas e iniciativas subregionales pre-existentes y el principio fundamental de que la prioridad de los países miembros del bloque es la consolidación del Mercosur el cual en ningún caso debe verse debilitado o diluido por los acuerdos que el Alca puede alcanzar.

Puede afirmarse de que a partir de 1996 con la firma de los acuerdos de asociación con Chile y con Bolivia y en particular a partir de la reunión de Belo Horizonte de 
1997 el Mercosur gana en madurez y proyección como bloque en la gestión y determinación con que maneja unitariamente sus políticas exteriores. Esta dimensión se recordará es formalmente consagrada en el Protocolo de Ouro Preto de diciembre de 1994 con la asunción de personalidad jurídica plena por parte del Mercosur, primera reforma de gran envergadura del Tratado fundacional del Mercosur de 1991 (Por su articulación Interna del Arancel Externo Común y su política comercian común aún imperfecta o incompleta).

Esta articulación externa cobra mayor cuerpo no sólo en la gimnasia de diálogo, negociación y culminación de acuerdos de cooperación y de libre comercio del Mercosur con otros bloques económicos (la Unión Europea, el Tlcan, la Comunidad Andina, el Mercado Común Centroamericano, los países del CER (Australia y Nueva Zelanda) y Japón.; sino que se refuerza a partir de las respuestas a las propias crisis internas. Los sucesos que como es de dominio público amenazaron la estabilidad democrática de Paraguay a partir de abril de 1996 vienen también a dar mayor fuerza al compromiso democrático del Mercosur (Mandato de San Luis de 1996, refrendado a mediados de 1998 en Ushuaia con plena fuerza jurídica) y a fortalecer los mecanismos de coordinación política y de temas de seguridad a partir de 1996 en el denominado Mecanismo de Concertación Política del Mercosur o Mercosur Político.

El principal acuerdo alcanzado hasta el momento en el marco del Alca (refrendado en las reuniones mantenidas este año por el Comité de Negociaciones Comerciales del Alca en febrero en San José de Costa Rica y a mediados de año en Buenos Aires) reside en que el acuerdo constitutivo del Alca dé lugar a un "single undertaking". Es decir un tratado internacional que comprenderá todos y cada uno de los temas en discusión por los grupos de trabajo que las partes deberán aceptar en su totalidad. Se rechaza por tanto el enfoque de un early harvest (cosecha temprana) preconizado por Estados Unidos y que consiste en sancionar y ejecutar aquellos acuerdos previos o interinos que se vayan alcanzando en determinadas áreas. Ello sin perjuicio de que conforme a lo acordado en Miami, a propuesta argentina, los países asuman el compromiso de "avanzar sustancialmente" en la negociación hacia el año 2002.

Asimismo, se plantea también fijar un calendario preciso para la eliminación de las barreras no arancelarias y acordar la elaboración de un inventario de prácticas distorsionantes tales como el recurso a los créditos de exportación en este ámbito. Los países del Mercosur han dejado claramente de manifiesto que no aceptarían que ningún sector quedara excluido de las negociaciones y han defendido que la normativa que se apruebe en ámbitos tales como antidumping o normas sanitarias 
o fitosanitarias sean reglas acordadas dentro del Alca y en ningún caso se acepte el recurso a leyes nacionales como ha hecho en alguna ocasión Estados Unidos. Se rechaza asimismo el recurso a legislaciones sobre subsidios y dumping como cobertura a la imposición de barreras para-arancelarias en velada advertencia al uso habitual de este instrumento por Estados Unidos.

Los países del bloque fijaron su posición sobre nuevos puntos de la agenda negociadora con ocasión de la reunión del Comité de Negociaciones Comerciales del Alca celebrada en Buenos Aires en junio de 1998. Allí el Mercosur expuso su posición común sobre la práctica totalidad de los temas de la agenda: política de competencia; subsidios, antidumping y derechos compensatorios, acceso a mercados, servicios, compras gubernamentales, propiedad intelectual y solución de controversias.

La dinámica del Alca, con desarrollos aún inciertos en tanto el Congreso estadounidense no conceda la facultad de negociación rápida al Presidente para negociar acuerdos comerciales, hecho que no se producirá al menos tras la renovación parcial efectiva del Congreso. A principios de 1999, se ha acentuado el factor acelerador de la armonización de políticas entre los socios. Ello es especialmente notorio en el ámbito de servicios y compras gubernamentales. Pero es particularmente visible en su impacto sobre la adopción de posturas negociadoras comunes del bloque ante el exterior. El Mercosur ha rechazado la oferta negociadora de Estados Unidos consistente en impulsar desde un inicio la desgravación arancelaria, descartando negociaciones paralelas y promoviendo una negociación prioritaria sobre temas tales como facilitación de las condiciones de acceso para la inversión extranjera y otras áreas nuevas de la OMC, como comercio de servicios, compras gubernamentales, propiedad intelectual, patentes, medio ambiente y dumping laboral. Ante esta pretensión, el Mercosur propone una negociación escalonada y paso a paso en fases que dificultó en primer instancia el entendimiento con Estados Unidos más favorable a la simultaneidad.

Los diferentes estilos o técnicas de los negociadores entre Estados Unidos y América Latina han sido en muchas oacasiones causas de desentendimiento (Tortora 1999). Mientras que los primeros definen su negociación en función de temas y en segundo lugar en función de socios o foros económicos, los segundos en cambio se orientan en general a proceder de la manera inversa, es decir que definen su posición en función del foro o de la contraparte y luego los objetivos en el tema a negociar. A los Estados Unidos este estilo le permite avanzar en sus negociaciones en "espiral". Por este estilo, logra que una posición negociadora obtenida en una 
instancia bilateral o multilateral, la proponga luego como piso y techo de cada negociación siguiente, y así, sucesivamente continua subiendo el piso y el techo. Además cuando está logrando sus objetivos, le agrega en ocasiones como en el Tlcan, nuevos temas.

En el Alca su percepción es que este está por encima de las normas de apertura comercial global (bienes y servicios) acordados en la Ronda Uruguay, por encima del Tlcan, y al menos al mismo nivel de lo previsto en la CAN y el Mercosur. Es en este orden de ideas que Manuela Tortora sostiene que, algún día el Alca pasaría a ser una referencia para las negociaciones con los países asiáticos y luego en el ámbito multilateral de la OMC. El carácter preferencial que pudiera significar el Alca tendería a reducirse a medida que se finalizan acuerdos con otras regiones.

Las reuniones de Ministros de comercio y del Foro Empresarial de las Américas a mediados de mayo de 1997 en Belo Horizonte, marcaron la conclusión del "ciclo brasileño" en el proceso de negociación del Area de Libre Comercio de las Américas (Alca).

El evento fue ampliamente divulgado por la prensa del Mercosur, con énfasis en las divergencias entre la posición del bloque y las propuestas de los Estados Unidos. Sin embargo, una evaluación equilibrada de las reuniones sugiere que el proceso de negociación alcanzó avances significativos en las cuestiones esenciales y que hasta las divergencias y conflictos pueden estar ofreciendo una contribución a la construcción de mayores convergencias y nuevos espacios de cooperación fundamentales en un proceso de construcción de largo aliento como es el Alca.

En Belo Horizonte fueron ratificadas las convergencias alcanzadas en las reuniones anteriores, definiendo una trayectoria de construcción del Alca que atiende, en sus principios y objetivos, los intereses del Mercosur. La prominencia del Alca entre los acuerdos de integración existentes en el Continente fue afirmada explícitamente, al admitir su coexistencia con los demás acuerdos, "en la medida que los derechos y obligaciones, asumidos en estos acuerdos estén previstos, o no, en los derechos y obligaciones del Alca, o bien los sobrepasen" (Declaración Ministerial de Belo Horizonte).

La mayor contribución de la reunión de Belo Horizonte y del "ciclo brasileño" de las negociaciones para la construcción del Alca fue la afirmación, por el Mercosur, que el Alca no puede ser el resultado de una iniciativa unilateral (de los Estados Unidos) y que la lógica de la integración debe ser regida, no por la adhesión de los países de América Latina a una iniciativa de esta naturaleza, sino por la negociación 
entre países y bloques con intereses convergentes y divergentes. Varios analistas norteamericanos, entre ellos Henry Kissinger, parecen haber comprendido esto, al resaltar que "el desafío para la política de los Estados Unidos es tratar con el Mercosur como un componente vital para la estructura del Hemisferio Occidental" y que la construcción del Alca exige una alteración de algunos métodos y posturas tradicionales en las relaciones con los Estados Unidos con América Latina.

La reunión ministerial de Belo Horizonte fue marcada por una retórica de conflicto y divergencias entre los Estados Unidos y Brasil, pero en la práctica, una vez más ratificó las importantes convergencias alcanzadas.

Cuatro puntos de convergencia valen la pena subrayar:

Primero, en una definición más precisa de la fecha de inicio de las negociaciones, los Ministros concuerdam que "deberían ser iniciadas en Santiago, en abril de 1998 y recomendamos a los Jefes de Estado y de Gobierno que lo hagan en aquella oportunidad". Aunque ésta no sea una afirmación taxativa, la fórmula usada deja poco margen de duda respecto de la fecha de inicio de las negociaciones. Para ellos, los Ministros de Comercio de los países del Continente formularán, en la reunión de San José de Costa Rica, en marzo de 1998 “cómo se procederá a las negociaciones, incluyendo aspectos tales como sus objetivos, enfoques, estructura y localización”.

Segundo, en la definición de "estructuras organizacionales de transición" entre la fase de pre-negociación y la etapa negociadora, los Viceministros tendrán tres reuniones en Costa Rica, para preparar el encuentro ministerial de marzo de 1998, antecesor de la Cumbre presidencial de Santiago en abril. Por otro lado, los viceministros pasan a constituir un Comité Preparatorio coordinado y dirigiendo las actividades de los Grupos de Trabajo y recomendando a los Ministros el modo de "reconfigurar" los Grupos de Trabajo en grupos de negociación.

Tercero, la reafirmación de las necesidades específicas de las economías menores, en términos de "políticas internas adecuadas, asistencia técnica y cooperación, a fin de facilitar su participación efectiva en el proceso del Alca".

Cuarto, en la calificación otorgada a la coexistencia del Alca con los acuerdos bilaterales y subregionales entre países del Continente. En tanto prevalezca el principio de single undertaking como modelo de adhesión al Acuerdo, esta coexistencia será posible "en la medida que los derechos y obligaciones bajo tales acuerdos no estén previstos o excedan los derechos y obligaciones del Alca". 


\section{Evolución y desarrollo de las negociaciones}

En Estados Unidos, las resistencias al Alca asumen sobre todo la forma de presiones para la inclusión de los temas ambientales y laborales en la agenda de negociaciones, con vistas a una upward harmonization de las normas vigentes en América Latina. En Brasil las restricciones involucran esencialmente la negociación de acceso a mercados, tomando en cuenta las fragilidades estructurales de la industria reveladas por la liberalización comercial.

En el Plan de Acción aprobado en la Cumbre de Miami, además de fijar para el año 2005 la fecha para la conclusión de las negociaciones, los Jefes de Estado del Continente admitieron conforme lo expresado anteriormente, que el proceso del Alca avanzaría en "dos vías" y establecieron un cronograma de reuniones para acompañar el programa de trabajo definido.

En Denver, en junio de 1995, los Ministros de Comercio reafirmaron la coherencia del Alca con las reglas de la OMC y con los principios del regionalismo abierto. Se crearon siete Grupos de Trabajo, previéndose el establecimiento de otros cuatro en la reunión de Cartagena (marzo 1996).

La integración en dos vías, definida desde Miami, resguardaba los intereses de los países del Mercosur involucrados en un proyecto de unión aduanera, pero también preservaba para los Estados Unidos la viabilidad de la estrategia de extensión del Tlcan, inclusive como elemento de presión durante el proceso negociador.

En la reunión viceministerial de Florianópolis, por iniciativa del Mercosur, se abrió el debate sobre el proceso de negociación a concluirse en el año 2005. De acuerdo con la propuesta del Mercosur, el proceso de negociación sería conducido en tres etapas sucesivas, vinculándose indisociablemente un conjunto de temas a cada una de ellas.

Así, en la primera etapa se buscaría la concreción de acuerdos de facilitación de negocios, en campos tales como procedimientos aduaneros, medidas sanitarias, etc. En la segunda etapa se incluirían temas cuya negociación exigiese más que acuerdos de facilitación de negocios, sin implicar cambios de concesiones en términos de acceso a mercado.

Estas estarían reservadas a la tercera etapa a iniciarse en el año 2003. Inaugurada la discusión en torno del proceso de negociaciones, se agregaron cinco propuestas más a la del Mercosur durante las reuniones de Recife (febrero de 1997) y Río de Janeiro (abril de 1997): las de los Estados Unidos, Canadá, Caricom, Grupo Andino y Chile. 
Las propuestas del Mercosur preveían negociaciones en tres etapas y dos en el caso de los Estados Unidos. Posteriormente a la reunión de Recife, los Estados Unidos adoptaron el modelo de negociaciones simultánea de todos los temas. Como fue expresado, el cuadro que se consolidó en vísperas de la reunión de Belo Horizonte presentaba algunas importantes divergencias y convergencias:

- En el plano de las divergencias, la más importante oponía la propuesta del Mercosur a todas las demás e involucraba el ritmo y la secuencia de las negociaciones. Por un lado, el Mercosur defendía que la Cumbre presidencial, prevista para abril de 1998 en Santiago, marcara el anuncio del "lanzamiento" de las negociaciones, mientras que, en las demás propuestas, este evento establecería el inicio efectivo de las negociaciones. Por otro lado, solamente la propuesta del Mercosur preveía negociaciones por etapas, vinculando el tratamiento de ciertos temas a determinadas etapas. Todas las demás propuestas defendían el tratamiento simultáneo de todas las áreas de negociaciones (Veiga 1997);

- Menos explícita, pero también importante, es la divergencia entre las propuestas norteamericanas (Estados Unidos y Canadá) y la del Mercosur, en cuanto al nivel de alcance del Acuerdo: mientras que los primeros identifican el montaje de arreglos comerciales y de disciplinas hemisféricas al establecimiento de normas OMC-plus, el Mercosur rechaza una adhesión general a este tipo de compromiso;

- Finalmente, la inclusión de temas laborales y ambientales en la agenda hemisférica, propuesta por los Estados Unidos, rechazada por el Mercosur, que condiciona la inclusión de nuevos temas a la comprobación de su vinculación al comercio y a la "maduración" de su tratamiento en el plano multilateral.

A las convergencias ya alcanzadas en las reuniones anteriores, el "ciclo brasileño" de negociaciones agregó, hasta la reunión ministerial de Belo Horizonte:

- La admisión de la necesidad de una estructura de soporte permanente a las negociaciones, aún cuando hubiese divergencia en cuanto a las funciones específicas y a la organización de dicha estructura; $\mathrm{y}$

- El consenso sobre la importancia de la participación del sector empresarial en el proceso de negociación, marcada por la intensa movilización privada en torno de la preparación de la reunión de Belo Horizonte.

\section{Balance y perspectivas de las negociaciones del Alca}

En lo que va del proceso del Alca se lograron producir un conjunto importante de convergencias sobre puntos fundamentales de negociación. Más allá y a pesar de las resistencias domésticas (principalmente en los Estados Unidos y Brasil) el proceso muestra señales crecientes de irrevesibilidad y la intensa agenda de 
negociaciones intergubernamentales y de reuniones empresariales asegura una permanente movilización de los agentes públicos y privados en torno del Alca.

Aunque el componente conflictivo, presente en toda negociación, sigue influenciando las posiciones de los Estados Unidos y de Brasil en relación al Alca más de lo que sería deseable, la evolución reciente del proceso muestra expectativas optimistas en relación a la "despolarización" de las negociaciones y un énfasis creciente en la dimensión cooperativa en las futuras discusiones.

Por lo menos tres factores contribuyen para convalidar estas expectativas:

- La participación crecientemente activa de países, como Canadá y Chile y de grupos de países como el Caricom y la Comunidad Andina, amplían la calidad de los actores relevantes en las discusiones intergubernamentales; ya que estos dos grupos actúan cada uno, cada vez más como bloque con voz propia en las negoicaciones.

- La importancia cada vez mayor de las propuestas y de las negociaciones del sector privado, caracterizadas por un elevado grado de convergencia; y

- Los efectos que la propia dinámica de negociaciones complejas y multi-temáticas generan sobre la formación de las posiciones nacionales y de coaliciones entre países y bloques, induciendo la aparición de diversas "posiciones nacionales" dentro de cada país y de coaliciones temáticas y sectoriales entre países y bloques.

Otro factor de cambio de las negociaciones es el agregado de un nuevo actor que ya ha surgido en las negociaciones, la Sociedad Civil. Su forma de participación y representatividad no ha sido definida y dependerá de la forma en que lo instrumenten los gobiernos para que se transforme en un factor de dinamismo y facilitador o obstaculizador o de rechazo al proceso (Salazar 1998).

La convergencia de estos tres factores tiende a crear un cuadro favorable a la superación de situaciones de impasse y de polarización, típicas de procesos negociadores donde existen pocos actores relevantes.

El "mapeo" de los incentivos y costos potenciales de participación del Mercosur en el Alca y la identificación de los factores que pueden influir en el balance final de ganancias y pérdidas resultante del acuerdo permiten una evaluación de los resultados provisorios y de las perspectivas de las negociaciones del Alca para el Mercosur.

Dos verificaciones autorizan esta evaluación:

Hasta el momento, las convergencias obtenidas esbozan una trayectoria de construcción del Alca que atiende a los intereses del Mercosur. La consistencia con las reglas de la OMC y el principio del regionalismo abierto, la posibilidad de 
negociar en grupo y de hacer del Mercosur uno de los building blocks del Alca, así como la adopción del single undertaking, no solamente como modelo de adhesión al acuerdo, sino también como principios de generación de un balance equilibrado de deberes y obligaciones entre los países, son avances que distancian el proceso de la construcción del Alca de las propuestas de creación del mercado hemisférico por extensión del Tlcan.

Por los menos teóricamente, esta propuesta reduce los riesgos de desvío de comercio e inversión en detrimento del Mercosur y atenúa la amenaza de discriminación contra países extra-Alca. Estas conquistas no son, todavía, irreversibles. La concesión del fast track por el Congreso al Ejecutivo de los Estados Unidos puede “resucitar" el modelo de extensión del Tlcan, particularmente si se mantuviera el impasse entre los Estados Unidos y el Mercosur sobre la secuencia de las negociaciones. Por otro lado, solamente la puntualización de normas comerciales y de inversión, índices de contenido y reglas de origen permitirá confirmar la inexistencia de una faceta anti-terceros países en el acuerdo hemisférico.

Las perspectivas de la posición negociadora del Mercosur en el Alca estarían condicionadas por la capacidad del bloque para contribuir a la superación de los impasses remanentes en la fase pre-negociadora.

La posición negociadora del Mercosur tiene como trasfondo una doble verificación: los países del bloque son global traders y por lo tanto, la asociación preferencial con los Estados Unidos no se caracteriza como una integración "natural" entre países con elevado grado de interdependencia económica. Además, los riesgos de la integración son elevados en función de la acentuada asimetría entre las economías potencialmente involucradas.

Es importante resaltar que la percepción de costos y beneficios que organiza la llamada "posición Mercosur" está supeditada ampliamente a la visión dominante en el gobierno brasileño acerca del proyecto Alca. El punto fuerte no contingente en la posición del Mercosur es su poder de negociación en este proceso, dada la importancia del bloque dentro de América Latina como mercado de destino de las exportaciones e inversión externa de los Estados Unidos y la elevada participación del PBI del bloque en el total de América Latina.

Sin embargo, también las fortalezas y fragilidades de las posiciones de negociación tienen un componente contingente. En este caso, el Mercosur tiene entre los puntos fuertes de su posición negociadora, la legitimidad política que disfruta la postura de cautela de los gobiernos en relación al ritmo y a la secuencia 
de las negociaciones, teniendo en vista el apoyo recibido de las asociaciones empresariales y de los sindicatos de trabajadores de la subregión, en Belo Horizonte.

Por lo que respecta al comercio entre el Mercosur y Estados Unidos, cabe señalar que las exportaciones del Mercosur hacia EE.UU crecieron un $20 \%$ en el período 1990-97, pasando de 9.600 a 11.500 millones de dólares, mientras las importaciones lo hicieron en un $275 \%$, pasando de 5.600 a 21.150 millones de dólares. En materia de inversiones, Estados Unidos acapara el $40 \%$ de los flujos de IED.

Estados Unidos acumuló en 1997 un superávit superior a los 8.000 millones de dólares frente al Mercosur lo cual es altamente significativo mientras su déficit comercial global alcanzaba su mayor pico en la década de los 90 con 113.700 millones de dólares. Ello tiene como correlato una pérdida de porción del mercado en Estados Unidos de los dos socios mayores del Mercosur. Las exportaciones argentinas a Estados Unidos pasaron de representar un 13,8\% del total en 1990 a un 7,8\% en 1997 y en el caso de Brasil pasó del 24,6\% al 17,5\%. Asimismo, Estados Unidos se convertía en el primer inversor extranjero en Argentina superando a la UE con el $36 \%$ del total.

Proyecciones y estudios coinciden en que América Latina será en el 2010-2020 más importante para los Estados Unidos como mercado que Europa y Japón. Por otra parte, se puede apreciar como las exportaciones Europeas en esta década crecieron y pasaron de ser deficitarias a tener superávit con América Latina, pero no crecieron al acelerado ritmo que las de Estados Unidos. Según fuentes oficiales, en el Año 2010 las exportaciones de Estados Unidos a la región se duplicarían en valores reales a los de mediados de los noventa y excederían el conjunto de ventas a Europa y Japón (Informe de Irela 1997).

El incentivo representado por la ampliación, sobre bases estables, del acceso al mercado de los Estados Unidos merece en el caso del Mercosur, dos calificaciones:

Por un lado, los países del Mercosur, con la excepción de Paraguay son como se ha mencionado global traders, o sea tienen importantes relaciones comerciales con diferentes regiones del mundo. En este sentido, para ninguno de ellos los Estados Unidos son un "socio comercial natural" y la mejora de las condiciones de acceso al mercado de ese país difícilmente generaría significativos impactos inmediatos sobre las exportaciones globales del Mercosur. Por lo tanto, la intensidad de este incentivo no debe ser sobrestimada, en el caso del Mercosur, principalmente en la medida que exista en el horizonte la perspectiva de nuevas negociaciones 
multilaterales, que desde el punto de vista de países global traders debilitaría la rationale para acuerdos regionales.

Por otro lado, las exportaciones de los países del Mercosur para los Estados Unidos especialmente las de Argentina y Brasil, son intensivas en manufacturas comparativamente a los patrones de ventas para la Unión Europea y Asia. La mejora de las condiciones de acceso al mercado de los Estados Unidos beneficiará los sectores productores de manufacturados y podría incentivar la expansión del comercio intra-industrial entre el Mercosur y los Estados Unidos. En este sentido la intensidad de este incentivo para los países del Mercosur tampoco debería ser subestimada.

\section{A modo de conclusión: Perspectivas}

En el actual contexto de turbulencias financieras y de otro orden podríamos afirmar que:

- la crisis en el Sudeste asiático, que aún no ha terminado, tiene efectos visibles en el freno a algunas iniciativas de liberalización y de integración en y con la región (ver como botón de muestra las abiertas discrepancias que se exhibieron en la cumbre de la Apec celebrada en Kuala Lumpur entre algunos países asiáticos y americanos) y la renuencia de Japón a atender las exigencias de apertura de su mercado agrícola, entre otras disensiones;

- la prolongada incertidumbre que pesa sobre la restauración de la estabilidad financiera y la credibilidad internacional de Brasil como país centro del contagio de la crisis rusa en América del Sur y en particular en el Mercosur;

- la hecatombe climatológica que se ha abatido sobre América Central, que entre otros efectos, podrá desviar la atención técnica y financiera de gran parte de los organismos multilaterales (en particular el BID) asociados a la formulación y apoyo a la negociación del Alca, al margen de comprometer la continuidad de los países centroamericanas en la participación al mismo ritmo que los restantes países americanos en la negociación del Alca.

Puede preverse, por consiguiente, un cierto parón en los meses próximos de la dinámica de progresión de los acuerdos comerciales a escala planetaria, con la única excepción hasta el momento de la Unión Europea que entra en su Unión Monetaria profundizando su integración hacia adentro pero como lo demuestran la complejidad de las negociaciones sobre la agenda 2000, la crisis de la Comisión Europea y la guerra en los Balcanes puede demorar su ampliación hacia fuera.

En todo caso, la dinámica de multilateralización y de profundización de los acuerdos comerciales recuperará su fuerza en cuanto queden conjuradas las actuales inestabilidades. 
El '99 se presenta como un año complejo e intenso en el numero de encuentros de los negociadores en el Alca pero no se esperan resultados muy significativos. Además de los efectos negativos de la crisis de Brasil en el primer semestre del año, varios factores apuntan a visualizar una perspectiva poco alentadora.

En primer lugar, el Alca del segundo semestre que expondrá sus resultados en la reunión de ministros de comercio en octubre en Canadá estará muy condicionada por los resultados de la reunión Ministerial de la OMC de noviembre donde se fijarán las futuras pautas de negociación en las nuevas rondas agrícolas y de servicios y una posible ronda más amplia conocida como la Ronda del Milenio.

En segundo lugar, en el segundo semestre del ' 99 y hasta pasando el primer semestre del año 2000 la actividad decisional hacia adentro y hacia afuera en el Mercosur se reducirá drásticamente por la situación pre y post electorales en la mayoría de sus miembros incrementando, las inseguridades provocadas por la crisis de Brasil y el Paraguay y comprometiendo el rumbo futuro. En este contexto será difícil que el Mercosur continúe fuertemente disciplinado hacia afuera como lo ha demostrando desde Belo Horizonte. Si aflora algún conflicto entre los dos grandes o entre los menos grandes, o los efectos de la crisis agrava la cohesión podría verse afectado el proceso de integración dando impulso a mayor bilateralidad.

La Comunidad Andina y Mercosur en sus iniciales negociaciones de principio de año no fueron esperanzadoras y no se espera grandes progresos antes de fin de año y no se vislumbra un fortalecimiento del bloque de América del Sur en las negociaciones del Alca.

En Europa la agenda 2000, que se compromete a acordar a mas tardar a fines de 1999 acuerdos tendientes a efectivizar la ampliación de la UE hacia el Este incluyendo sies países nuevos antes del 2005, en una primer etapa y poner en marcha el nuevo marca financiero presupuestario para el periodo 2000-2006 que incluye revisiones importantes de políticas como la agrícola que han provocado controversias importantes. Esta importante agenda sumado a los temas relativos al euro y las cuestiones internas producto de desencuentros interinstitucionales y un cercano cambio de las autoridades colegiadas de la Comisión, mantendrá este proceso con limitada vitalidad hacia fuera y tendrá posiblemente un efecto atenuador en las decisiones y proyectos que se quieran implementar en las relaciones Mercosur-Unión Europea, México-UE, en las más programáticas de la cumbre eurolatinoamericana de Río y así como en las relaciones transatlánticas Estados Unidos- Europa lanzadas en 1998. 
En el Tlcan, hacia adentro en 1999 continuaran acelerándose el ritmo de los programas de desgravación arancelarias iniciados el ano anterior, sin embargo en su desarrollo hacia afuera no se espera el ingreso de nuevos miembros ni negociaciones entre bloques antes que termine el siglo, dada las dificultades en obtener la vía rápida para la administración Clincton absorbida en parte por un prematuro clima pre electoral.

El ritmo de los acuerdos bilaterales de libre comercio continuaran con el ritmo actual fortaleciendo la telaraña de acuerdos bilaterales y convergiendo y facilitando el comercio a nivel hemisférico a pesar de esta conyuntura, la integración múltiple y simultanea continuará en el próximo siglo como parte de una dinámica global desordenada.

La dinámica inter-temporal y secuencial gravitará sobre la marcha de los inéditos acuerdos de comercio Norte-Sur o diagonales (UE-Mercosur; Tlcan-Mercosur a través del Alca, Apec). El ritmo diferenciado de consecución de los acuerdos, dependerá sin duda de una multiplicidad de factores: mayor sintonía política y de políticas, gestión de los contenciosos inter e intra-regionales en forma directa o a través de las nuevas disciplinas de la OMC, comportamiento de la inversión y de las diferentes "regionalidades" de origen de las multinacionales más dinámicas. El lanzamiento del euro constituye un nuevo desafío en el próximo siglo para la carrera regional a escala planetaria en el tejido de las alianzas comerciales y financieras y ya se puede apreciar un importante debate sobre las monedas únicas o la dolarizacion en todos los regionalismos.

Es en el marco de esta nueva dinámica mundial que se esta gestando al inicio del próximo siglo que un Triángulo Atlántico, Mercosur “Ampliado" o Alcsa, América del Norte y Europa podría competir con el alicaído Triángulo Asia Pacífico, América de Apec.

Numerosos acontecimientos ya empiezan a demostrar la valides de esta hipotesis. Las nuevas Cumbres presidenciales y los renovados dialogos no nacen por acto espontaneo sino en el marco de una creciente competencia entre regiones. Cumbres Hemisféricas, Cumbre Eurolatinoamerica, Cumbre Asia pacífico, Cumbre Euro-asiática. Estas reflejan movimientos de los bloques, alianzas y otras articulaciones de un mundo multipolar en formación pero en el que aun, algunos polos son más centrales que otros.

Las ideas de trilateralidad que primaba en los 70 y 80 entre Estados Unidos, Europa y Japón como ejes del poder mundial en un contexto de conflictos Norte- 
Sur y Este-Oeste han cambiado radicalmente en los 90. Los bloques mas o menos definidos han sustituido esa ya vieja lectura y las alianzas entre estos bloques en relaciones horizontales, verticales y diagonales pasan a ocupar un lugar fundamental dada la escala planetaria de los acontecimientos económicos, financieros, políticos y de seguridad.

Varios triángulos de distinta intensidad parecen reemplazar la trilateralidad pasada:

Por un lado, Europa juega sus diagonales con Asia, y América Latina sin dejar de lado su lado Atlántico con Estados Unidos. Estados Unidos juega sus diagonales con el Asia Pacífico y América Latina sin dejar de lado su lado Atlántico con Europa. Y América Latina con Estados Unidos y Europa sin dejar de lado su lado Asia Pacífico.

Consideraciones como estas, despiertan interrogantes sobre como será el orden internacional del siglo XXI y las perspectivas de competencia y cooperación, de divergencias y convergencias. En este heterogéneo escenario mundial el triángulo Atlántico $^{2}$ entre América Latina, Norteamérica y Europa se presenta en términos relativos homogéneo y con una red de acuerdos ya existentes y de futuro próximo que construyen grandes avenidas y un entramado de gran solides. Un mercado futuro de más de 1200 millones y una historia política, económica, religiosa y cultural, percepciones y modelos sociales y valores compartidos nos unen mucho más que otras regiones del mundo a pesar de las diferencias. Es un espacio de competencia y cooperación, de divergencias y convergencias único en el mundo por su similitud en los "códigos de conducta".

La competencia entre Europa y Estados Unidos sobre una región de emergente crecimiento y gran potencial económico como América Latina, segunda de mayor crecimiento en el mundo, es una realidad muy actual y futura. Para América Latina mantener una relación diversificada que sirva de contrapeso entre dos grandes polos de atracción a político y cultural es muy importante y históricamente ha estado presente en toda la joven historia de estas repúblicas, y ella lleva 175 años si contamos desde la doctrina Monroe en 1824.

Mantener una equidistancia entre estos dos polos no ha sido, no es y no será un camino facil para la region Latinoamérica. Las diferencias existen también al interior de cada bloque y las formas de vincularse entre estos subbloques en el

\footnotetext{
${ }^{2}$ Informe de IRELA, 1997.
} 
triángulo. Este es un componente importante y muchas veces ha sido determinante en el momento de mantener una estrategia de relacionamiento que perdure en el largo plazo.

La Teoría del péndulo basada en que las discrepancias entre los polos del norte amplía el poder de negociación del sur es relativa. Los riesgos de ser prisionero de esta teoría como único estilo de relacionamiento con los otros dos polos del triángulo es importante ya que en los temas muy candentes o cuestiones mayores no ha funcionado. El Triángulo debe y tiene que mantenerse abierto.

Sin embargo la competencia, como la cooperación así como las divergencias y las convergencias son y están en movimiento constante y son procesos que se desarrollan en múltiples direcciones. Las posiciones cambiantes de las partes, la instabilidad estructural y la asimetría de poder existente en las relaciones, cuestiona el aun centro hegemónico del triángulo a pesar de compartir con éste una importante comunidad de intereses.

No es mera coincidencia que la Cumbre eurolatinoamericana recae bajo presidencia alemana, que tiene gran interes en la región y que en 1997 en la Reunión de Noordwijk entre la UE y el grupo de Río sostenia su ministro la posibilidad de hacer converger los procesos de acercamiento de Europa, estados Unidos y América Latina en una gran Area de Libre Comercio Transatlántica (Alcta).

Otro triángulo es el que se desarrollara entre las negociaciones que tendrán lugar en al OMC, la integración subregional y el Alca. Si las dudas aumentan frente a la factibilidad del Alca muchos se preguntan que sin posibilidad de una "vía rápida" en el corto plazo, una ronda del milenio cercana y las incrementales resistencias de algunos sectores al libre comercio y la globalización se acentúan por la crisis financiera y esta se globaliza y afectará la dinámica de los mercados, la tasa de crecimiento y el comercio mundial.

Si no tiene éxito los esfuerzos de coordinación macroeconómica para evitar una recesión global, un nuevo ciclo de estilo proteccionista puede marcar en forma desfavorable los proceso de integración en el hemisferio y la economía mundial de inicios del próximo siglo. Si ello ocurre y riesgos existen tendremos en lugar de un triángulo Atlántico de los más prósperos del mundo un simple y nada llano triángulo de las Bermudas. 


\section{Bibliografía}

Documentos Sela (1998). El Papel de Canadá en el Hemisferio. n²8-29, out.

Grabendorff, Wolf e Riordan Roett (1984). América Latina, Europa y Estados Unidos. ¿Un nuevo Triángulo Atlántico? Buenos Aires: Grupo Editor Latinoamericano.

Grandi, Jorge (s. d.). Hacia una sociedad civil del Mercosur. Revista Integración y Comercio. Buenos Aires: Intal/BID.

Grandi, Jorge (s. d.). Déficit y déficit democrático y social en espacios integrados. Revista Integración y Comercio. Buenos Aires: Intal/BID.

Informe de Irela (1997). ¿Un desafío al Triángulo Atlántico? Contexto y agenda de una cumbre UE-América Latina. Madrid, maio.

Krenzler, Horst G. e Gunnar Wiegand (1999). European Foreign Affairs. Journal, Leicester University, Sais Review, Washington D. C: John Hopkins University, jun.

Reidy, Joseph (1964). Latin America and the Atlantic Triangle, Orbis. 1: p. 52

Salazar, José Manuel (1998). Estado y perspectivas de las negociaciones del Alca, OEA Unidad de Comercio. Miami, out. (Presentación ante la Red Empresarial para la Integración Hemisférica).

Tortora, Manuela (1999). Alca: expectativas y realidades. Home Artículos y Discursos del Sela, Caracas, fev.

Veiga, Pedro da Motta (1997). Revista Integración y Comercio. Intal/BID. sept-dic. 
